\title{
Supporting Information: Nanodiffraction Imaging of Polymer Crystals
}

\author{
Shusuke Kanomi ${ }^{1}$, Hironori Marubayashi ${ }^{2}$, Tomohiro Miyata ${ }^{2}$, Kenji Tsuda ${ }^{3}$, and Hiroshi Jinnai ${ }^{* 2}$ \\ ${ }^{1}$ Department of Applied Chemistry, School of Engineering, Tohoku University, 6-6 Aramaki Aza Aoba, Aoba-ku, Sendai, Miyagi 980- \\ 8579, Japan. \\ ${ }^{2}$ Institute of Multidisciplinary Research for Advanced Materials, Tohoku University, 2-1-1 Katahira, Aoba-ku, Sendai, Miyagi 980-8577, \\ Japan. \\ ${ }^{3}$ Frontier Research Institute for Interdisciplinary Sciences, Tohoku University, 6-3 Aramaki Aza Aoba, Aoba-ku, Sendai, Miyagi 980- \\ 8578, Japan.
}

\section{Detailed measurement conditions for WAXD/SAXS.}

Simultaneous wide-angle X-ray diffraction (WAXD) and small-angle X-ray scattering (SAXS) measurements were performed at the beamline BL-6A $(\lambda=0.1500 \mathrm{~nm})$ of the Photon Factory at KEK (Tsukuba, Japan). The beam size was $200 \mu \mathrm{m} \times 500 \mu \mathrm{m}$ in the vertical and horizontal directions, respectively. The divergence angles of the X-ray beam in the vertical and horizontal directions were evaluated to be 0.046 and 0.30 mrad, respectively. Two-dimensional (2D) WAXD and SAXS patterns were detected using PILATUS 100K and PILATUS3 1M (DECTRIS Ltd.), respectively. The samples were maintained at room temperature during the measurements. Silver behenate was used as a standard for both WAXD (007-0012) and SAXS (001). Characteristic spacings were extracted from the SAXS data using correlation function analysis. ${ }^{44,45}$ A laboratory-scale WAXD apparatus (D8 ADVANCE, Bruker) equipped with a 2D detector (PILATUS3 R 100K-A, DECTRIS Ltd.) was used to evaluate the degree of crystallinity and crystal orientation. A parallel-mirror-monochromatic X-ray beam $(\lambda=0.1542 \mathrm{~nm}, \mathrm{Cu} \mathrm{Ka})$ was collimated to a diameter of $0.5 \mathrm{~mm}$ just before the sample. Silicon powder was used as the standard. A series of X-ray structure analyses were performed using handmade software. ${ }^{56,57}$

\section{Results of WAXD/SAXS/SAED measurements.}

Detailed description of the WAXD results. Figure S1a, b shows the 2D WAXD patterns of the non-oriented and oriented films, respectively. The 110 and 200 reflections of orthorhombic $\mathrm{PE}^{35}$ are clearly visible in both samples. The non-oriented film exhibited a ring pattern generated by randomly oriented crystalline lamellae, whereas the oriented one showed spots indicating the well-oriented crystalline lamellae (i.e., the $c$ axis) parallel to the stretching direction. The crystallinities of the non-oriented and oriented films were calculated as 73.7 and $65.2 \%$, respectively, using the peak separation method. ${ }^{65}$ The orientation degree of the oriented sample was evaluated as $93.9 \%$ from the azimuthal full width at half-maximum [FWHM $\left.\left(^{\circ}\right)\right]$ in the 110 peak [i.e., $\left(180^{\circ}-\mathrm{FWHM}\right) / 180^{\circ}$ ]. Thus, it was confirmed that both non-oriented and oriented samples exhibited high crystallinity $(\approx 70 \%)$, and lamellar crystals in the oriented sample were strongly aligned in the stretching direction (i.e., row structure).

Detailed description of the SAXS results. Figure S1c, d depicts the SAXS patterns of the non-oriented and oriented films, respectively. The nonoriented film showed a ring pattern formed from randomly oriented lamellar stacks, whereas the oriented film showed a two-point scattering characteristic of oriented lamellar stacks in which the stacking direction is parallel to the stretching direction. The WAXD crystallinity exceeded $50 \%$; hence, longer and shorter spacings could be assigned to $l_{c}$ and $l_{\mathrm{a}}$, respectively, in the SAXS correlation function analysis. ${ }^{44}$ For the nonoriented film, the long period $\left(L_{\mathrm{p}}\right)$, lamella thickness $\left(l_{\mathrm{c}}\right)$, and amorphous layer thickness $\left(l_{\mathrm{a}}\right)$ were calculated to be 20.6 , 16.1, and $4.4 \mathrm{~nm}$, respectively, where $L_{\mathrm{p}}=l_{\mathrm{c}}+l_{\mathrm{a}}{ }^{44,45}$ For the oriented film, $L_{\mathrm{p}}, l_{\mathrm{c}}$, and $l_{\mathrm{a}}$ were $13.6,9.5$, and $4.1 \mathrm{~nm}$, respectively. The linear crystallinities $\left(l_{\mathrm{c}} / L_{\mathrm{p}}\right)$ were evaluated to be 78.4 and $69.7 \%$ for the non-oriented and oriented samples, respectively. 

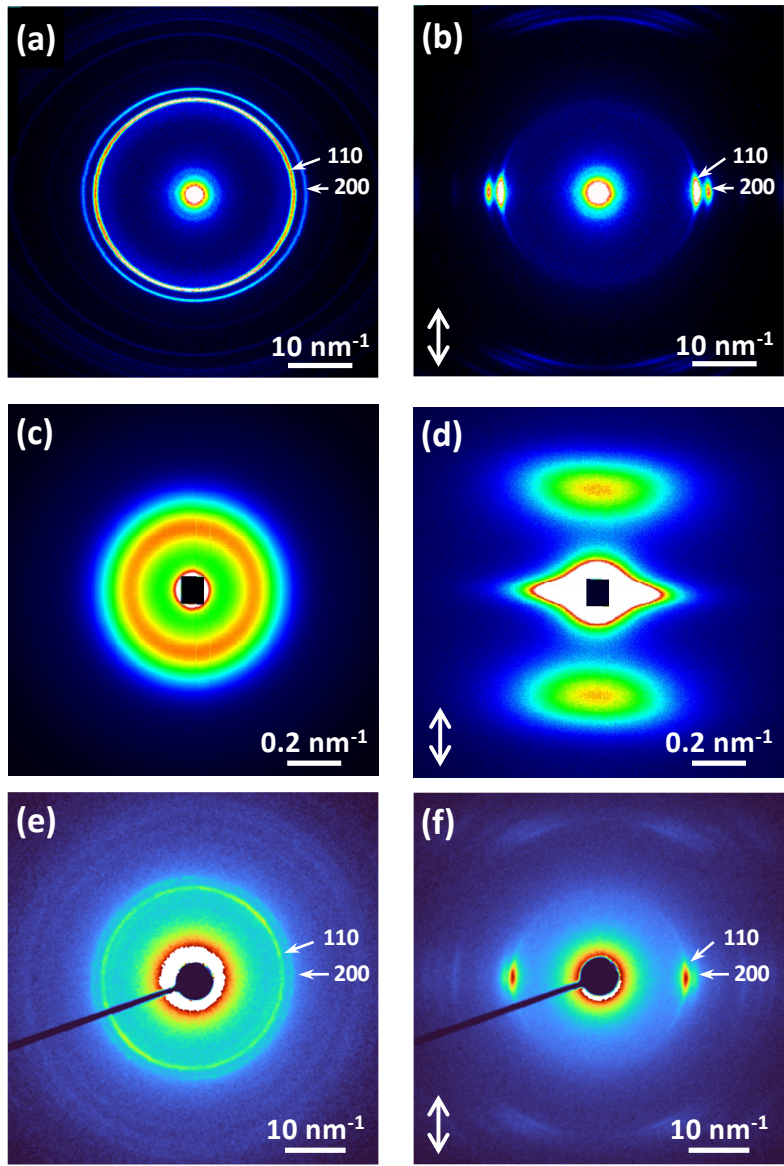

Figure S1. (a, b) Wide-angle X-ray diffraction, (c, d) small-angle X-ray scattering, and (e, f) selected-area electron diffraction patterns of (a, c, e) nonoriented and (b, d, f) oriented polyethylene. The arrows in (b), (d), and (f) indicate the stretching direction. All scale bars denote constant values of the scattering vector $q(=4 \pi \sin \theta / \lambda)$.

Hierarchical structure of polyethylene.

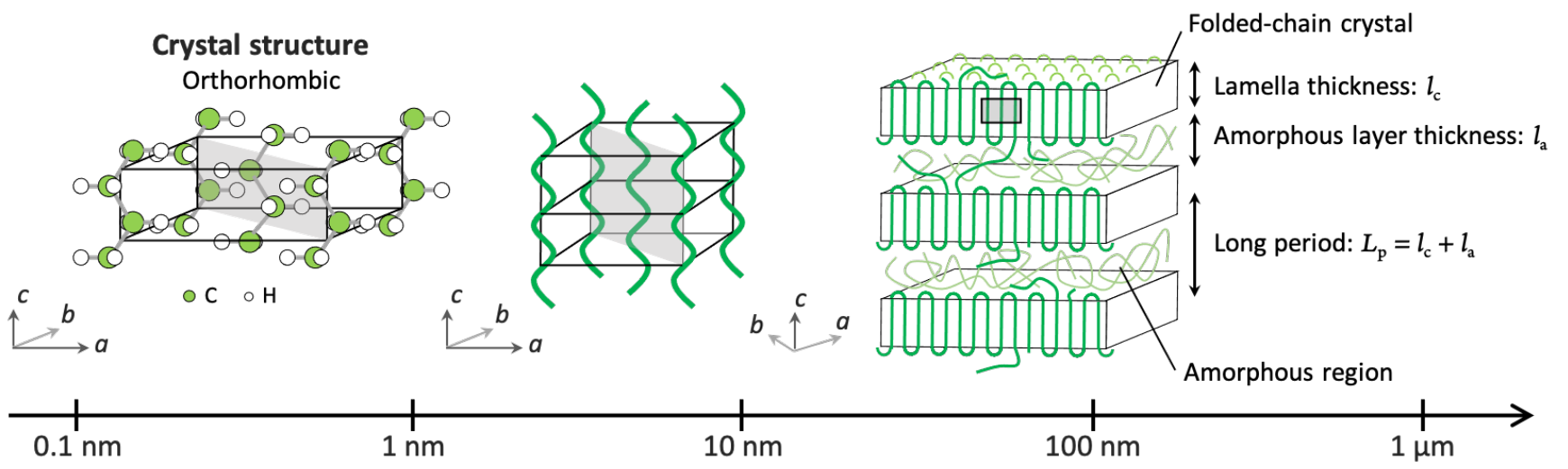

Figure S2. Schematic illustration of the hierarchical structure of polyethylene. 
Electron irradiation dose. Previous studies have presented only the dose per unit area; however, we also show the dose rate (dose divided by exposure time). The methods of estimating or measuring the values (flux or beam current) used to calculate the doses differ. We measured the number of electrons per second using a OneView camera.

Table S1. Comparison of experimental nanodiffraction imaging parameters for beam-sensitive organic materials.

\begin{tabular}{|c|c|c|c|c|}
\hline Author & Panova et al. & Panova et al. & Gallagher-Jones et al. & Present work \\
\hline Year & 2016 & 2019 & 2019 & 2021 \\
\hline Sample & P3HT/PS blend & PBTTT & Peptide & Polyethylene \\
\hline TEM & FEI Titan & FEI TitanX & TEAM I & JEOL JEM-F200 \\
\hline Voltage $[\mathrm{kV}]$ & 200 & 300 & 300 & 200 \\
\hline Detector & Gatan Orius & Gatan Orius & Gatan K2 IS & Gatan K3 IS \\
\hline Temperature $[\mathrm{K}]$ & 77 & 77 & 77 & 296 \\
\hline Convergence angle [mrad] & 0.51 & 0.48 & 0.5 & 0.2 \\
\hline Probe diameter $[\mathrm{nm}$ at FWHM $]$ & 7 & 2 & 6 & 15 \\
\hline Dwell time $[\mathrm{s}]$ & 0.07 & 0.033 & 0.0025 & 0.02 \\
\hline Step size $[\mathrm{nm}]$ & 20 & 10 & 20 & 30 \\
\hline Flux $\left[\mathrm{e}^{-} / \mathrm{s}\right]$ & - & - & $1.5 \times 10^{6}$ & $8.7 \times 10^{6}$ \\
\hline Beam current $[\mathrm{pA}]$ & 5 & 5 & - & - \\
\hline Dose per probe area $\left[\mathrm{e}^{-} / \AA^{2}\right]$ & 600 & 3000 & 1.3 & 10 \\
\hline Dose per scan area $\left[\mathrm{e}^{-} / \AA^{2}\right]$ & 50 & 100 & 0.094 & 1.9 \\
\hline Dose rate per probe area $\left[\mathrm{e}^{-} / \AA^{2} \mathrm{~s}\right]$ & 8100 & 100000 & 530 & 510 \\
\hline Dose rate per scan area $\left[\mathrm{e}^{-} / \AA^{2} \mathrm{~s}\right]$ & 780 & 3100 & 38 & 96 \\
\hline
\end{tabular}

Damage evaluation based on ED patterns and averaged profiles. It is known that electron irradiation damage increases with increasing dosage when all other conditions are held constant. The effect of damage on the electron diffraction (ED) can be evaluated by repeatedly scanning the same field of view.
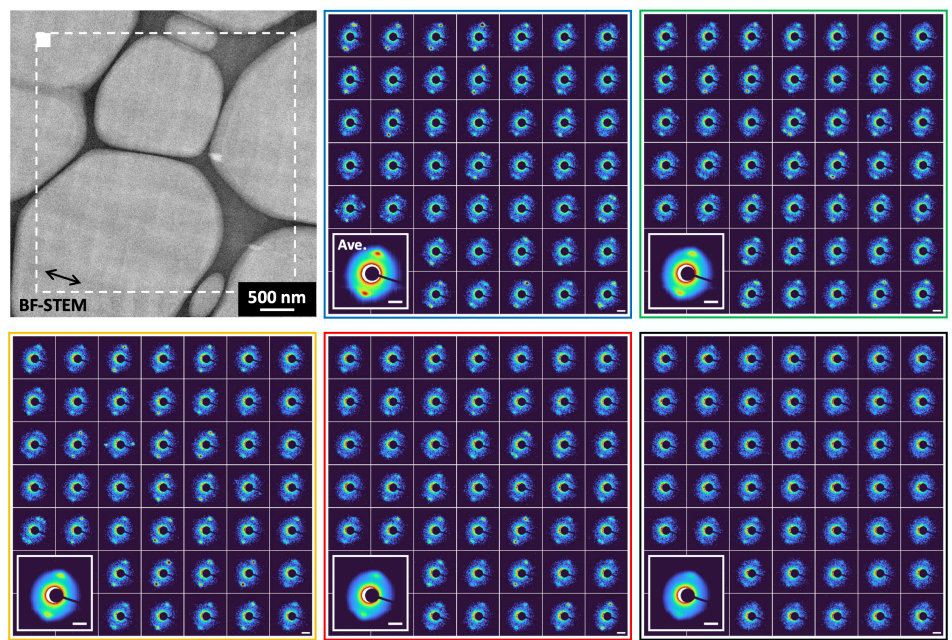

Figure S3. Exposure time (proportional to the electron dose) dependence of electron diffraction (ED) patterns in nanodiffraction imaging (NDI). The $128 \times 128$ scan was performed in the area enclosed by the dashed white frame [bright-field scanning transmission electron microscopy (BF-STEM)] under the conditions described in the paper, and the $7 \times 7$ ED patterns of the first scan at the upper left-hand side of the scanning area (white square) are shown in the blue frame (upper middle). The insets depict an averaged ED pattern. The green, yellow, red, and black frames indicate the ED patterns from the second, third, fourth, and fifth scans, respectively. All scale bars in ED patterns are identical $\left[10 \mathrm{~nm}^{-1}\right.$ in $\left.q(=4 \pi \sin \theta / \lambda)\right]$. The black arrow in the BF-STEM image indicates the stretching direction. 
In the case of beam-scanning techniques such as nanodiffraction imaging (NDI), we must determine whether the electron irradiation damage induced by the first scanning step affects the subsequent step. Measurements were performed with different step sizes ( $5,10,15$, and $30 \mathrm{~nm})$ under identical conditions. The ED intensity decreased with number of scans, even when the step size ( $30 \mathrm{and} 15 \mathrm{~nm})$ was equal to or larger than the beam diameter ( $15 \mathrm{~nm}$ in FWHM), indicating that damage also occurred outside the beam diameter. When the step size (10 and 5 $\mathrm{nm}$ ) was smaller than the beam diameter ( $15 \mathrm{~nm}$ in FWHM), the damage to the following scanning areas became more significant.
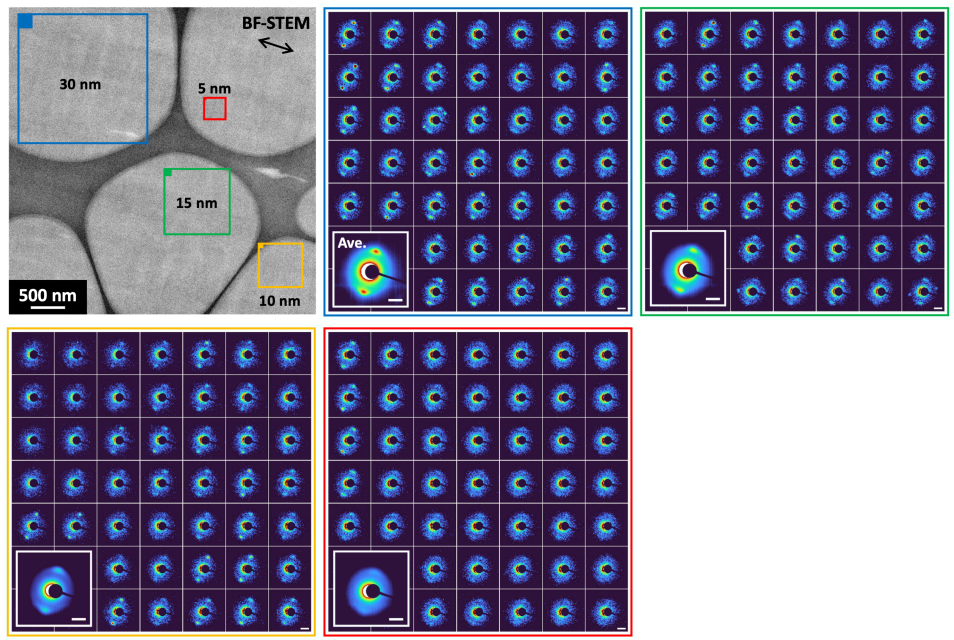

Figure S4. Step-size dependence of electron diffraction (ED) patterns in nanodiffraction imaging (NDI). The $64 \times 64$ scan was performed within the blue frame [bright-field scanning transmission electron microscopy (BF-STEM)] under the conditions described in the paper (i.e., a step size of 30 $\mathrm{nm}$ ), and the $7 \times 7 \mathrm{ED}$ patterns at the upper left-hand side of the scanning area (blue square) are shown in the blue frame (upper middle). The insets depict an averaged ED pattern. The green, yellow, and red frames indicate areas scanned at step sizes of 15, 10, and $5 \mathrm{~nm}$, respectively. All scale bars in the ED patterns are identical $\left[10 \mathrm{~nm}^{-1}\right.$ in $\left.q(=4 \pi \sin \theta / \lambda)\right]$. The black arrow in the BF-STEM image indicates the stretching direction.

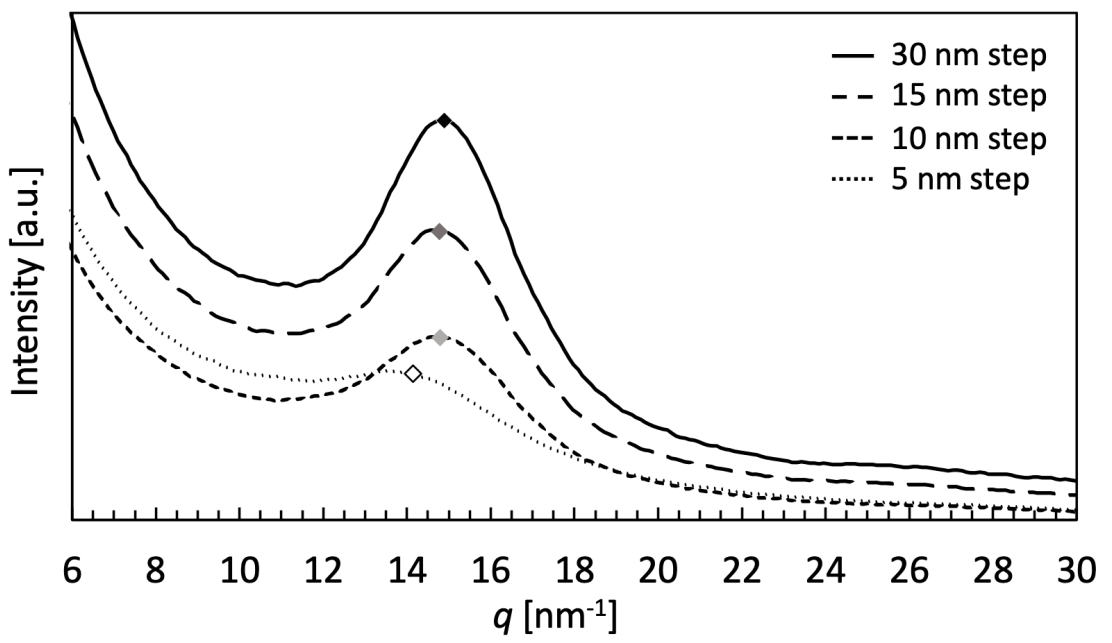

Figure S5. Step-size dependence of sector-averaged scattering profiles of averaged electron diffraction patterns. By reducing the scan step from 30 to 5 $\mathrm{nm}$, the irradiation dose per scan area $\left(D_{\mathrm{s}}\right)$ was increased from 1.9 to $70 \mathrm{e}^{-} / \AA^{2}$. 
Tiles of all ED patterns in NDI. Each ED pattern was cropped and arranged according to the scanning position of the electron beam.

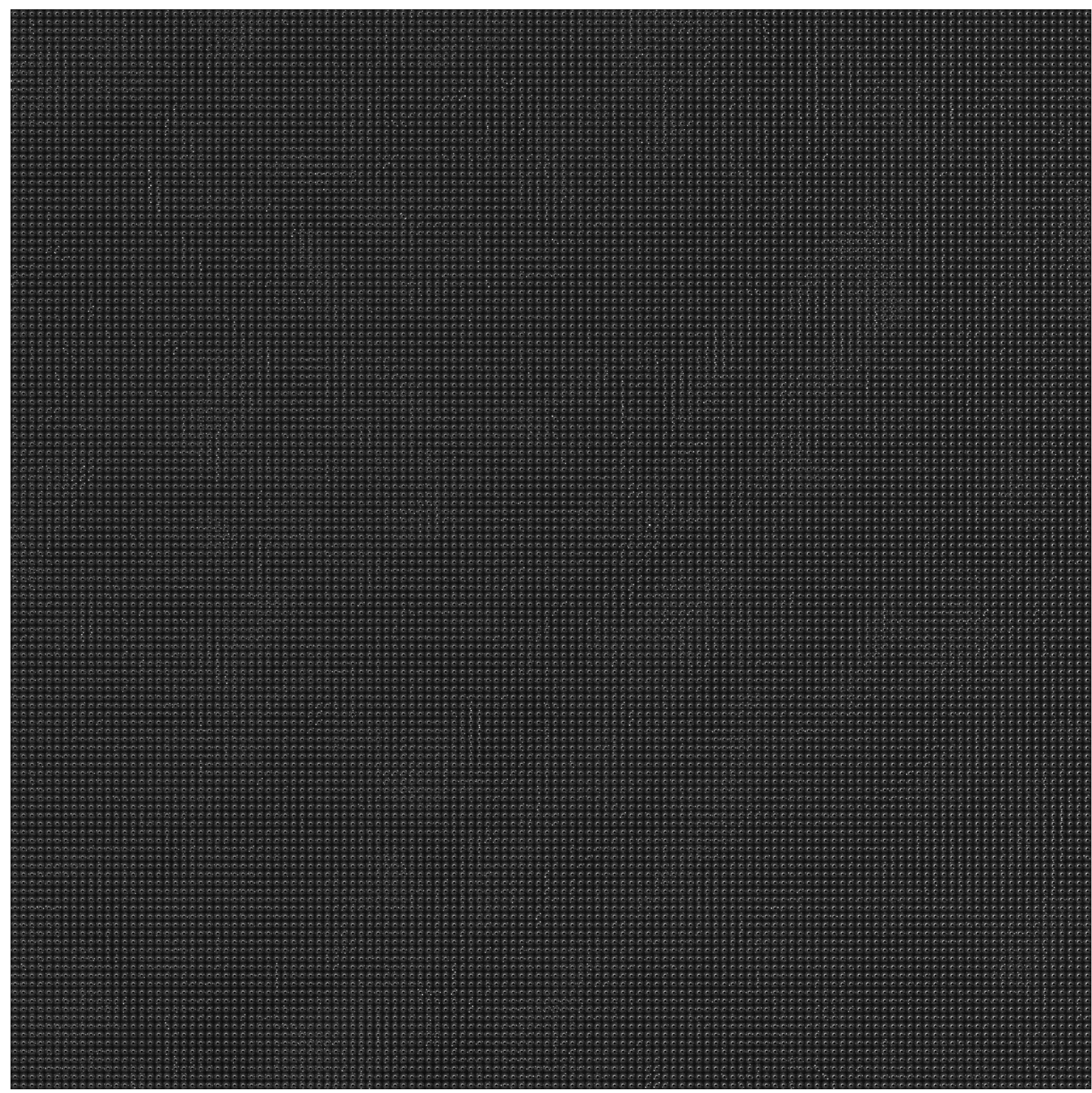

Figure S6. Electron diffraction pattern tile $(128 \times 128)$ of non-oriented polyethylene. 


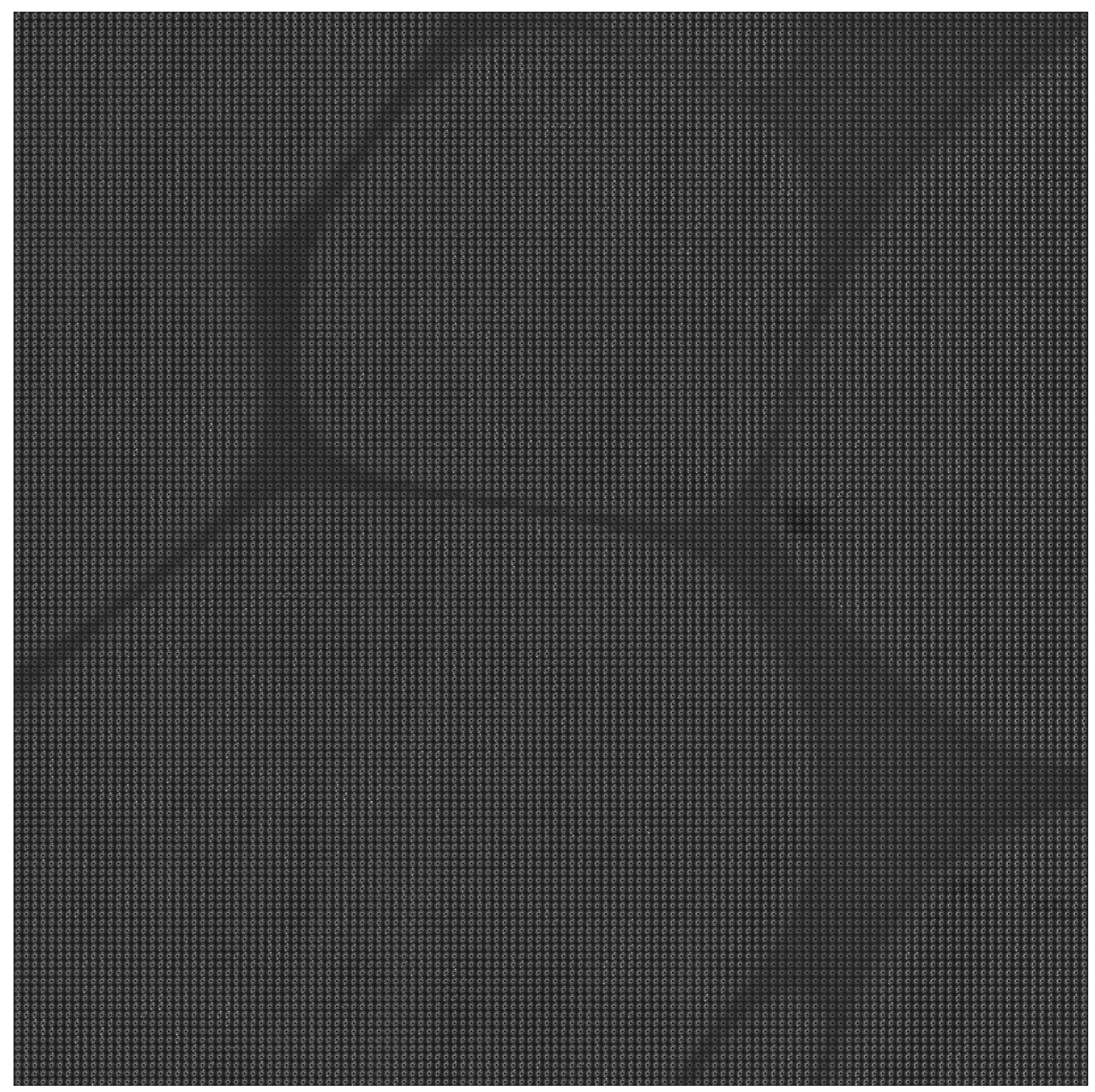

Figure S7. Electron diffraction pattern tile $(128 \times 128)$ of oriented polyethylene. 
The statistically-averaged ED patterns of the total NDI scanning region $(3.84 \mu \mathrm{m} \times 3.84 \mu \mathrm{m})$ were calculated by averaging $16384(=128 \times$ 128) ED patterns (Figure S8). The averaged ED pattern of the non-oriented sample exhibited a weak ring pattern (Figure S8a), whereas that of the oriented sample showed 2-spot diffraction (Figure S8b). These results are in qualitative agreement with those of WAXD and SAED (Figure S1). Because the total scanning area of NDI is comparable to the selected area of SAED ( $3 \mu \mathrm{m}$ in diameter), consistency between the averaged NDI and SAED is expected. However, in reality, the ring and spots in the averaged ED patterns are broader than in the WAXD and SAED patterns. The primary reason for this quantitative disagreement is that the electron beam in the NDI was a convergent beam, whereas the SAED used a parallel beam. The stability of the electron microscopy scanning optics is a minor reason.
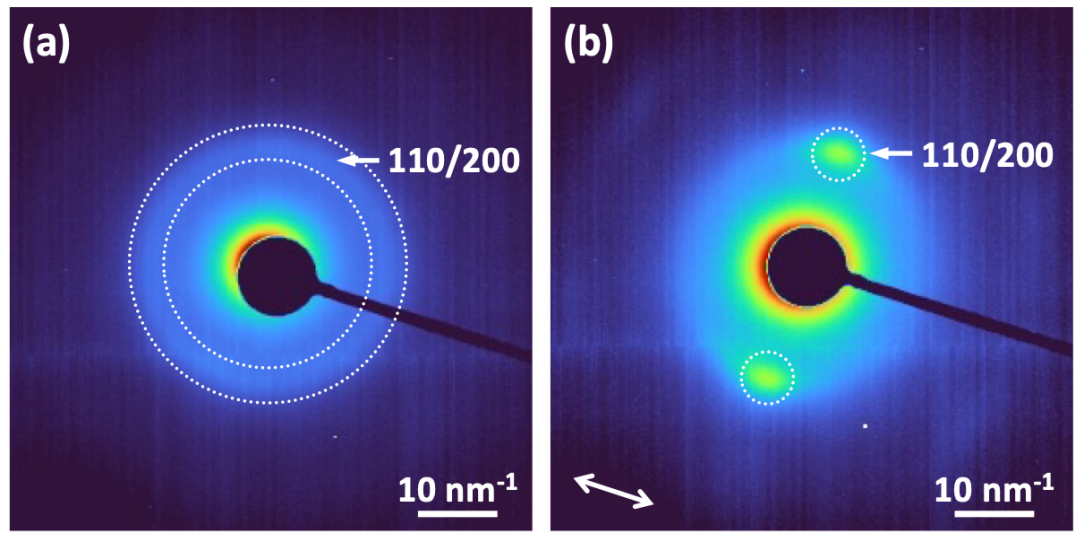

Figure S8. Averaged diffraction patterns of all $128^{2}$ diffraction patterns from (a) non-oriented and (b) oriented polyethylene. The double-headed arrow in (b) indicates the stretching direction. All scale bars indicate a constant value $\left(10 \mathrm{~nm}^{-1}\right)$ of scattering vector $q$.

Peak detection and map creation processes. (1) Initial peak position decision. Each ED pattern is very noisy (low signal-to-noise ratio); hence, weak peaks are buried in the noise. To remove noise and emphasize the peak, a process called "difference of Gaussians" was performed by taking the difference between Gaussian-filtered ED patterns with different standard deviations $\sigma$ (3 and 25). For the initial peak positions $\left(P_{\text {ini }}\right)$, we used the local maxima satisfying the following two conditions: (i) an intensity exceeding the 98.5 percentile of the histogram, and (ii) a sufficient distance from the center of the averaged ED pattern.

(2) Calculation of the center of gravity (COG). The COG was calculated within a circle region of radius 8 pixels $\left(1.7 \mathrm{~nm}^{-1}\right.$ in $\left.q\right)$, and the center was one of the $\mathrm{P}_{\mathrm{inis}}$. The COG calculation was repeated until the COG stopped moving. The final COG was defined as the final peak position $\left(\mathrm{P}_{\text {fin }}\right)$. COG calculations around other $\mathrm{P}_{\mathrm{ini}}$ were performed in a similar manner, and other $\mathrm{P}_{\text {fin }} \mathrm{s}$ were determined.

(3) Calculation of peak azimuth, distance, and intensity. The peak azimuths and distances were initially calculated using the center of the averaged ED pattern. Note: these are tentative values, because we know that the actual center of each ED pattern moves by a maximum of 15 pixels. The average peak intensity was calculated in a circular region of radius 8 pixels, and the center was one of the detected peak positions $\left(\mathrm{P}_{\text {fin }}\right)$. For the background, we used the average intensity of a torus region of inner and outer radii 8 and 10 pixels, respectively, and the center was $P_{\text {fin. }}$ The background intensity was subtracted from the average peak intensity. The background-subtracted average intensity was used as the peak intensity $(I)$.

(4) Calculation of ED pattern center. For this calculation, we used peaks satisfying the following two conditions: (i) the peak could be assigned to 110 or 200 reflections according to the distance from the center, and (ii) the azimuthal angle difference $(\Delta \mu)$ from the maximum intensity peak $\left(\mathrm{P}_{\text {fin, } \max }\right)$ was $\sim 0^{\circ}$ or $180^{\circ}\left( \pm 15^{\circ}\right)$. For the new center, we used the midpoint between the average peak position with $\Delta \mu \approx 0^{\circ}$ and that with $\Delta \mu \approx 180^{\circ}$. Re-calculations of the peak azimuth and distance were performed with the new center. These four processes were computed for all ED patterns.

The azimuth of $\mathrm{P}_{\mathrm{fin} \text {, max }}$ was used to create an orientation map. Because the peaks observed in the ED patterns of PE are $h k 0$, the azimuth of the peaks can be converted to that of the molecular chain (the azimuth of the molecular chains is perpendicular to that of the $h k 0$ peaks). The orientation map was created by plotting $\Delta \mu$ between the stretching and chain directions with respect to the scanning positions. The relationship between the azimuth and color is defined by the color wheel presented in the inset of Figure $7 f$. 
Distribution of lamellae orientation. Histograms of orientation maps were created to evaluate the distribution of chain orientation (the lamellae stacking direction was parallel to the chain direction). The histogram of the non-oriented sample was flat, indicating that the distribution of the chain orientation was random. In contrast, the histogram of the oriented sample featured a sharp peak $\left(\mathrm{FWHM:} \sim 10^{\circ}\right)$ at $\Delta \mu \approx 0^{\circ}$. This is in good agreement with the azimuthal FWHM obtained by WAXD $\left(10.7^{\circ}\right.$ and $\left.11.4^{\circ}\right)$.

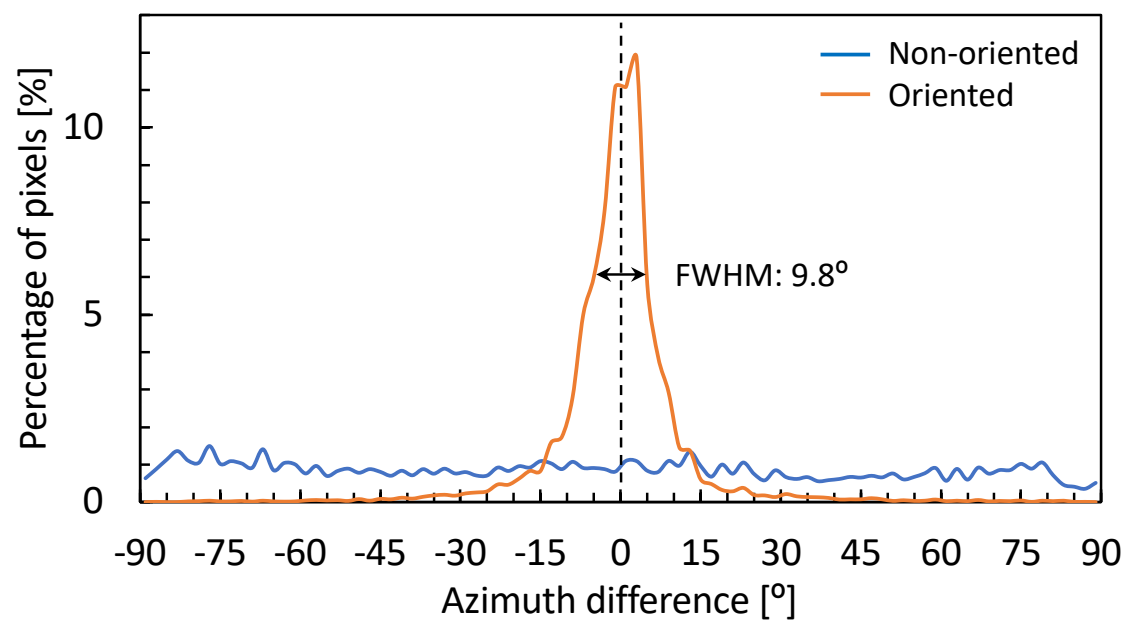

Figure S9. Histograms of orientation maps for non-oriented (blue) and oriented (orange) polyethylene.

Relative crystallinity map. The relative crystallinity map (Figure S10) was constructed by plotting the peak intensity ( $I$, see "Peak detection and map creation processes") with respect to scanning position. The intensities were normalized to the maximum value of each map.
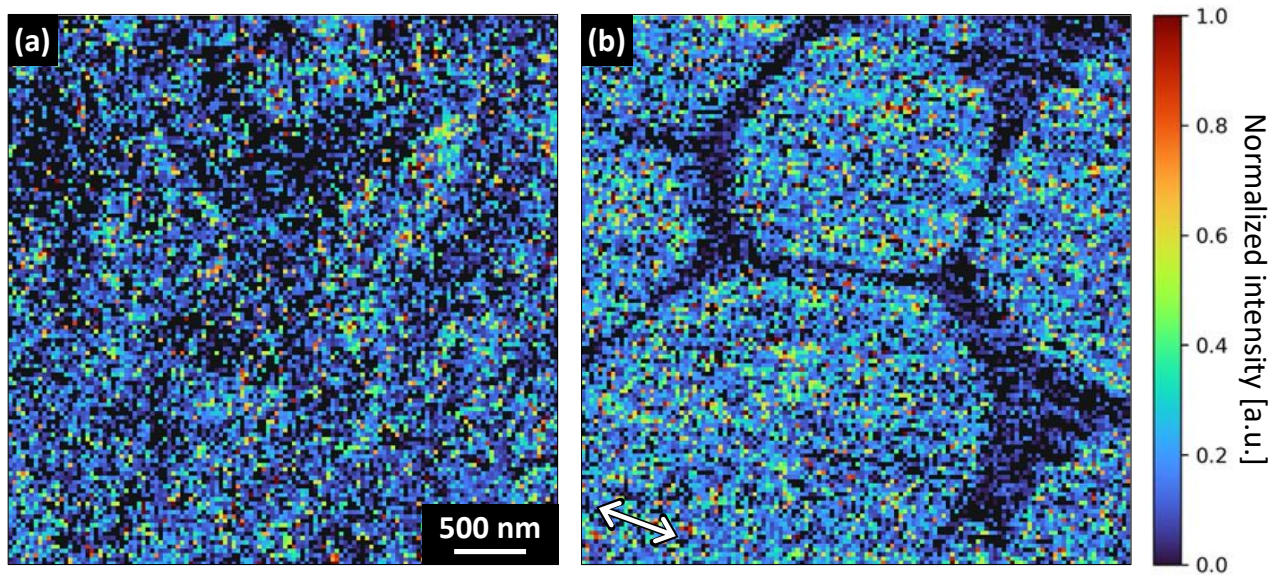

Figure S10. Relative crystallinity maps for (a) non-oriented and (b) oriented polyethylene. The intensities of each pixel are normalized by the maximum intensity of each map. The scale bar is identical between (a) and (b). The double-headed arrow in (b) indicates the stretching direction.

Analytical error. The errors in analytical methods of each ED pattern in NDI as the factors affecting the accuracy of orientations and relative crystallinity. There is the error of a few pixels in determining the peak positions by the center-of-gravity (COG) searching method. In NDI, we used the convergent electron beam that makes the diffraction spot size larger as compared to the parallel beam setting in SAED. In addition, intensity distribution within such broadened spot is somewhat complicated. Hence, the circle-area size for COG searching gives a few pixels error $\left(\epsilon_{\mathrm{COG}}\right)$. In addition, the center of ED pattern was determined by using two peak positions, so that the center position should also have a few pixels error $\left(\epsilon_{\text {center }}\right)$. Therefore, the crystal orientation will be influenced by both $\epsilon_{\mathrm{COG}}$ and $\epsilon_{\mathrm{center}}$, and the relative crystallinity will be affected by $\epsilon_{\mathrm{COG}}$. 
Damage visualization. In the case of $\mathrm{PE}$, it is possible to capture the appearance of electron irradiation damage by utilizing the intensity attenuation of ED spots. Figure S11 shows the electron-dose dependence of the relative crystallinity maps. When the dose was increased, the crystallinity of PE was markedly decreased. This result indicates that the same field of view should be scanned only once with the dose we apply here $\left(D_{s}=1.9 \mathrm{e}^{-} / \AA^{2}\right)$. Thus, the dose dependence of the relative crystallinity maps can be used as a measure of the irradiation damage.
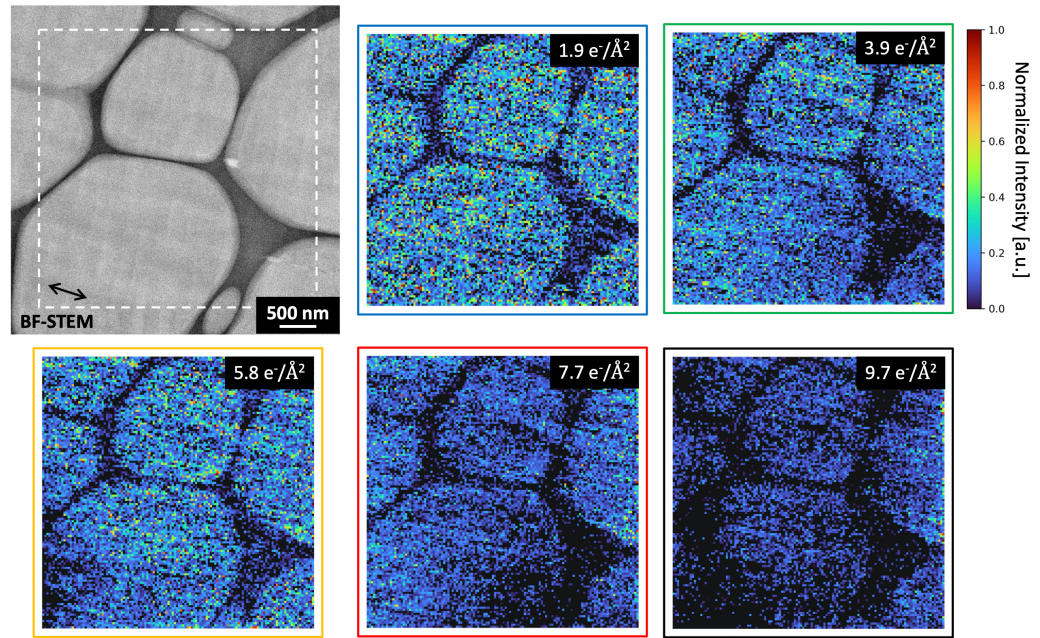

Figure S11. Electron dose (proportional to the electron beam exposure time) dependence of electron diffraction intensities. The $128 \times 128$ scan was performed within the dashed white frame under the conditions described in the paper, and the relative crystallinity map of the first scan is shown in the blue frame. The green, yellow, red, and black frames indicate the maps of the second, third, fourth, and fifth scans, respectively. The values of all pixels in each map are normalized by the same value. 